\title{
Comparison between Conventional and Improved Gas Engine-Driven VRF under Same Climate Conditions
}

\author{
Taku Shimizu ${ }^{1}$, Kyosuke Yamada ${ }^{1}$, Shigeki Kametani ${ }^{2}$ and Tatsuo Nobe ${ }^{1}$ \\ 1. Department of Architecture, Kogakuin University, Tokyo 163-8677, Japan \\ 2. Tokyo University of Marine Science and Technology, Tokyo 108-8477, Japan
}

Received: July 11, 2013 / Accepted: October 14, 2013 / Published: February 28, 2014.

\begin{abstract}
This paper reports the on-site performance evaluation of conventional and improved gas engine-driven VRF (variable refrigerant flow) units and (abbreviated as GHP) units. The study aims to elucidate two actual GHP units by using the probe insertion method. There is a tendency to decrease energy efficiency compared to a high loading factor. GHP operation was almost all part load operation. This on-site evaluation indicates a clear difference between conventional and improved GHP.
\end{abstract}

Key words: VRF system, the probe insertion method, GHP, APF.

\section{Introduction}

Recently, air-source VRF (variable refrigerant flow) systems have been adapted for use in small-and medium-scale office buildings. However, the actual performance of these VRF systems has never been fully substantiated. In this study, the authors have developed a measurement technique to evaluate the performance of a VRF system. The technique can be used in outdoor VRF units already installed on a building [1-4]. Using these techniques, the authors have measured VRF systems that are already installed on various buildings. A GHP (gas heat pump) air-conditioning systems of an air-conditioning unit use gas as its main energy source and operate with the involvement of a heat pump. GHP air conditioning has two advantages: (1) low power consumption due to the use of gas as the main energy source; (2) high-efficiency heating because a GHP system uses exhaust heat.

The subjects of this investigation were a GHPc (conventional type GHP) system and an GHPi

Corresponding author: Tatsuo Nobe, professor, research field: air-conditioning system and thermal comfort, E-mail: nobe@cc.kogakuin.ac.jp. (improved type GHP) system. GHPc systems are widely used. A GHPi unit is a device that considers the APF (annual performance factor) in a new evaluation method. The APF measures the efficiency of energy consumption throughout the year. The use of APF was proposed in 2006 by JIS (Japanese Industrial Standards). Values for APF in Japan are derived by considering the climatic conditions of Japan as a reference.

Fig. 1 illustrates the relationship between monthly average temperature and humidity in several countries. This graph was made with reference to "Weather underground" [5]. Japan experiences a much more

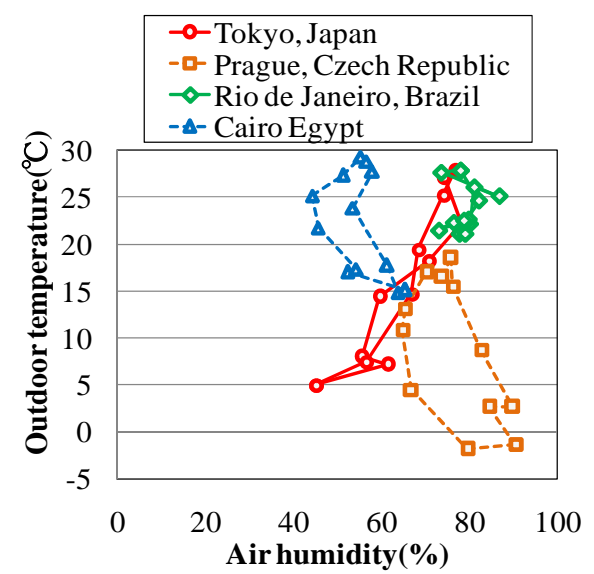

Fig. 1 Climograph by country. 
variable climate than that in many other countries; therefore, there is a demand for both cooling and heating. In such an environment, the rated COP (coefficient of performance) [6] is inappropriate for measuring the performance of a GHP system because the COP applies to an environment subjected to constant temperature. Therefore, the APF [7] has been introduced in Japan. This study aims to clarify the performance differences between the GHPc and GHPi system, and to assess the performance of the VRF systems.

\section{Method}

\subsection{Method of Computing Heat Exchange of the} Outdoor Unit

The authors measured the performance of the outdoor units of VRF systems already installed on buildings at different locations. Figs. 2 and 3 show the GHP. Using these energy-distribution diagrams and the income and expenditure equations (Eqs. (1)-(14)), the authors calculated the efficiency of the unit, which is expressed as the COP [4].

$$
\begin{gathered}
E_{P}=E_{E V} \\
E_{I G}=E_{T}+E_{X}+E_{R} \\
E_{T}=E_{I G}-E_{X}-E_{R} \\
E_{T}=E_{C D}-E_{E V} \\
E_{I G}-E_{X}-E_{R}=E_{C D}-E_{P} \\
E_{P}=E_{C D}+E_{R}+E_{X}-E_{I G} \\
C O P_{C}=\frac{E_{P}}{E_{I}}=\frac{E_{P}}{E_{I G}+E_{I G 2}} \\
E_{P}=E_{C D} \\
E_{I G}=E_{T}+E_{X}+E_{R}+E_{S} \\
E_{T}=E_{I G}-E_{X}-E_{R}-E_{S} \\
E_{T}=E_{C D}-E_{E V}-E_{S} \\
E_{I G}-E_{X}-E_{R}=E_{S}-E_{E V} \\
E_{P}=E_{E V}-E_{R}-E_{X}+E_{I G} \\
C O P_{H}=\frac{E_{P}}{E_{I}}=\frac{E_{P}}{E_{I G}+E_{I G 2}}
\end{gathered}
$$

\subsection{Heat Exchange Measurement of the Outdoor Unit}

Air that flows into the heat exchanger of the outside unit passes over the fins and then leaves the unit

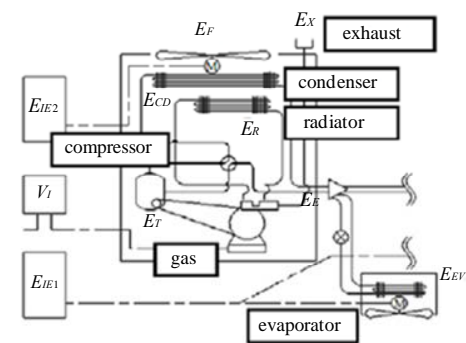

Fig. 2 Energy income and expenditure equation for GHP (cooling).

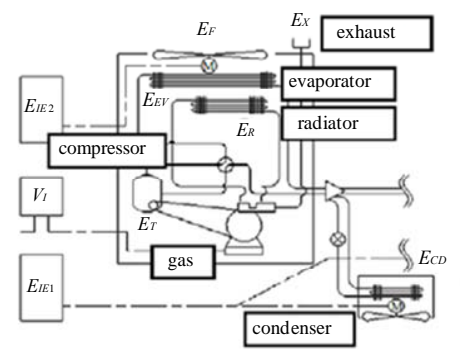

Fig. 3 Energy income and expenditure equation for GHP (heating).

through the fans. Part of the air that passes over the fins commutates and the heat exchangers change the air state quantity. In the probe insertion method, we calculated the heat exchanger efficiency (Eq. (15)) by measuring the difference between the air in the outside heat exchangers and that in the inside heat exchangers. Fig. 3 shows the measurement of the heat exchange in an outdoor unit.

$$
q_{a H}=\sum_{n=1}^{n} \alpha_{n} \times V_{F A N} \times S_{n} \times \times C_{P} \times\left(t_{\text {in.n }}-t_{\text {out. } n}\right)
$$

\subsection{Names and Unit}

Measurements used in Eqs. (1)-(15) are defined as follows: EI is the dissipation power; ET is the motive energy of the compressor; EF is the dissipation power of the fan; EEV is the heat quantity of the evaporator; ECD is the heat quantity of the condenser; EP is the heat exchanger efficiency; ER is the heat quantity of the radiator; EX is the exhaust heat quantity; COPC is the coefficient of the performance during air cooling; $\mathrm{COPH}$ is the coefficient of the performance during air heating; VFAN is the wind velocity of the fan; $S_{n}$ is the delegating area of the heat exchanger; $\rho$ is the air 


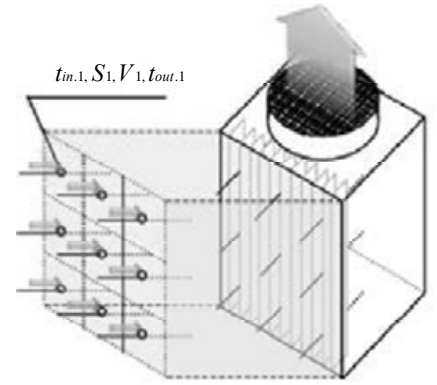

Fig. 4 Measurement of heat exchange in outdoor unit.

density; $C_{p}$ is the constant pressure specific heat; $t_{\text {out.n }}$ is the air temperature of the inside heat exchangers, and $t_{i n . n}$ is the air temperature of the outside heat exchangers; $\alpha_{n}$ is the wind velocity ratio.

\section{Survey Outline}

Table 1 presents a summary of the surveyed GHPs. The authors conducted measurements at an office building in Chuo-ku, Tokyo, over a period of one year from February 16, 2010 to February 15, 2011. There was almost no difference in the capacities of the GHPc and GHPi units, but the GHPi unit had higher gas consumption. Therefore, the GHPc unit had a higher COP. The authors confirmed that the performance of both the GHPc and GHPi units was higher in heating than in cooling.

Fig. 5 shows the operational status of each GHP. In April, air conditioning in Japan changes from heating to cooling, whereas the opposite occurs in November.

Figs. 6 and 7 illustrate the layout of the outdoor and indoor units, respectively. The GHPc outdoor unit was installed in the southeast of the building, while the GHPi outdoor unit was in the northwest. The GHPi indoor unit was installed in an office room towards the northwest, while the GHPc indoor unit was installed in an office room towards the southeast.

The GHPc indoor unit was installed in another room in which only one unit.

\section{Survey Results}

Fig. 8 shows the generated thermal energy for a year. Broken lines are for heating and solid lines are for cooling. Cooling demand is clearly much higher
Table 1 Summary of the surveyed GHP.

\begin{tabular}{|c|c|c|}
\hline & \begin{tabular}{|l}
$\begin{array}{l}\text { Improvement } \\
\text { type GHP }\end{array}$ \\
\end{tabular} & $\begin{array}{l}\begin{array}{l}\text { Conventional } \\
\text { type GHP }\end{array} \\
\end{array}$ \\
\hline & GHPi & GHPc \\
\hline Picture & & \\
\hline Location & Nihonbashi, T & yo, Japan \\
\hline Term & 2010/2/16-201 & $2 / 15$ \\
\hline Use building & Office & \\
\hline Cooling capacity & $56 \mathrm{kw}$ & $56 \mathrm{kw}$ \\
\hline Heating capacity & $63 \mathrm{kw}$ & $67 \mathrm{kw}$ \\
\hline Cooling COP & 1.17 & 0.96 \\
\hline Heating COP & 1.34 & 1.13 \\
\hline Cooling gas consumption & $45.1 \mathrm{kw}$ & $54.9 \mathrm{kw}$ \\
\hline Heating gas consumption & $44.3 \mathrm{kw}$ & $56.0 \mathrm{kw}$ \\
\hline $\begin{array}{c}\text { GHPi } \\
\text { - : heating } \\
\text { operation }\end{array}$ & $\begin{array}{l}\text { ooling }- \text { : heat } \\
\text { eration } \quad \text { opera }\end{array}$ & $\begin{array}{l}\text { HPc } \\
- \text { - cooling } \\
n \quad \text { operation } \\
\end{array}$ \\
\hline
\end{tabular}

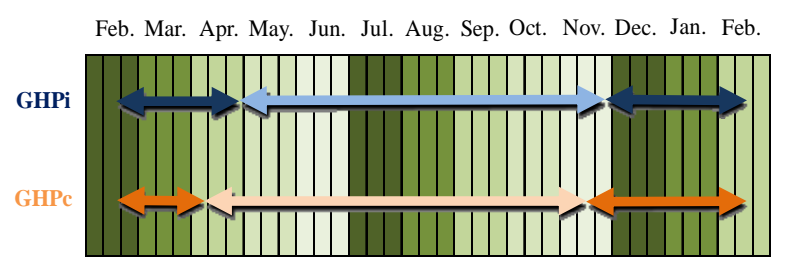

Fig. 5 Measurement of heat exchange in outdoor unit.

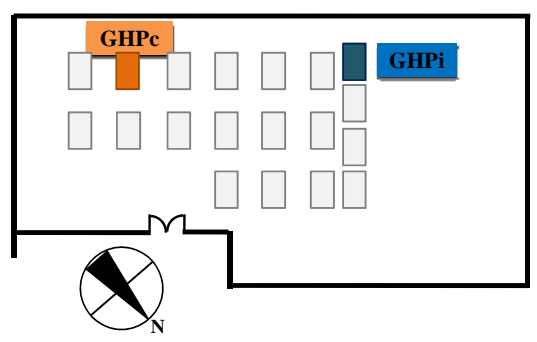

Fig. 6 Measurement of heat exchange in outdoor unit.

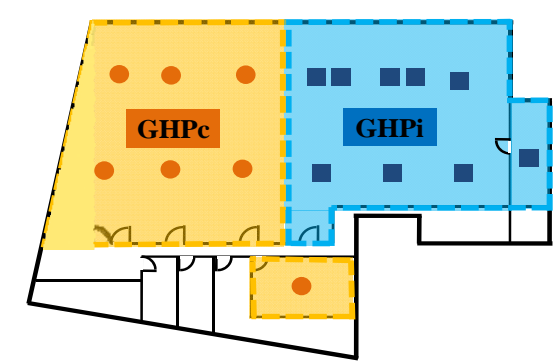

Fig. 7 Measurement of heat exchange in outdoor unit.

than heating demand. Furthermore, the GHPi experienced greater thermal demands than the GHPc. 


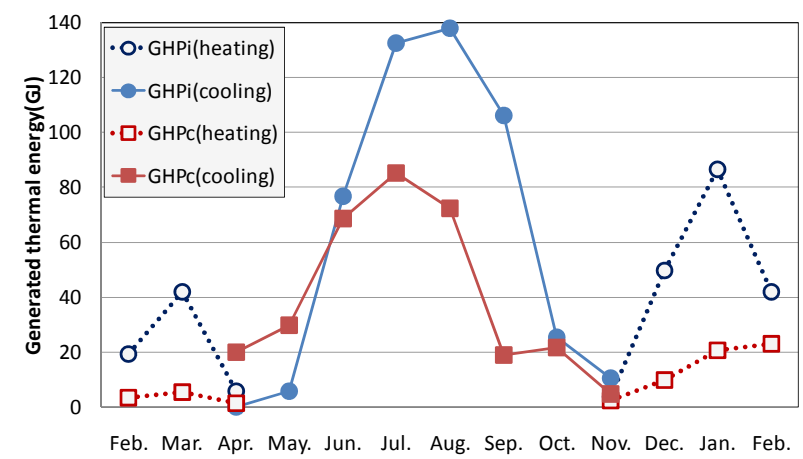

Fig. 8 Quantity of air changes in one year.

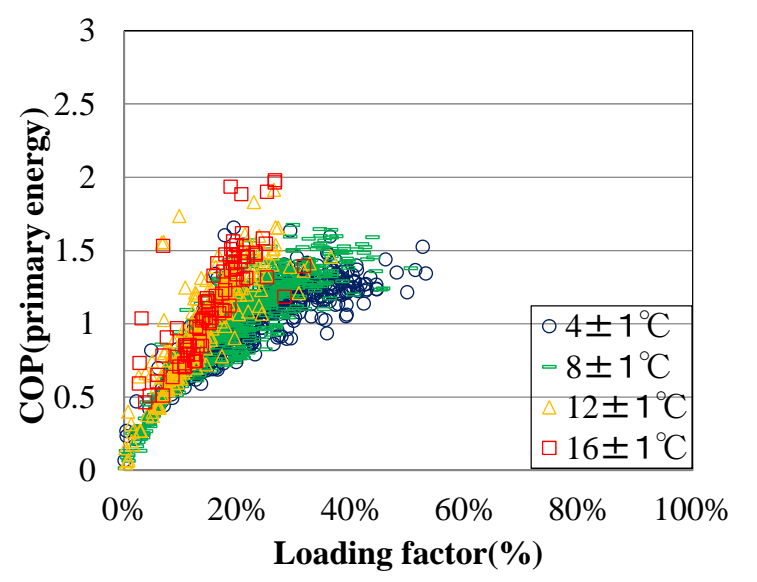

Fig. 9 Relationship between the COP and the loading factor (heating GHPi).

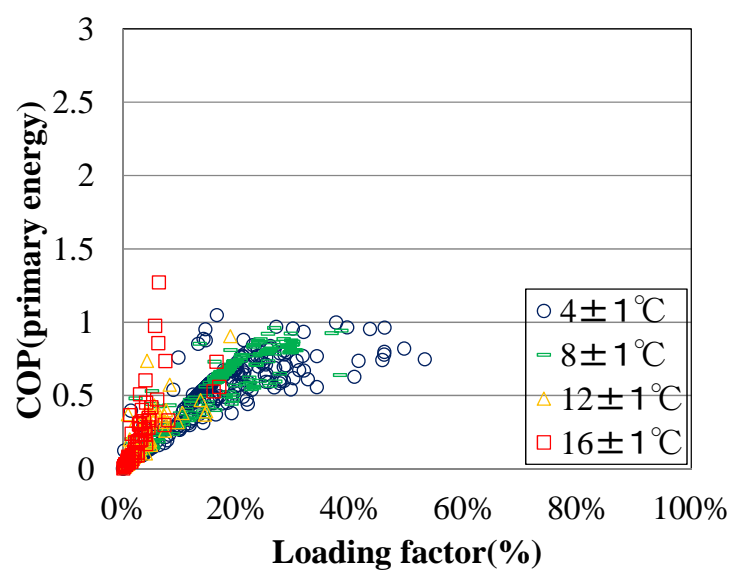

Fig. 10 Relationship between the COP and the loading factor (heating GHPc).

Figs. 9-12 show the relationship between COP and loading factor (the ratio of actual capacities to rated capacities), separated according to the outdoor temperature. During heating with the GHPc, the loading factor was around $40 \%$ or less and the loading factor was almost identical during cooling. During

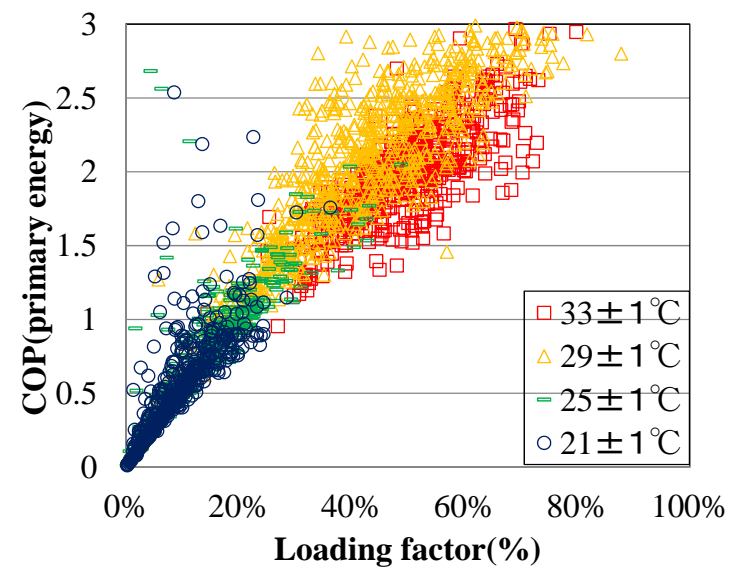

Fig. 11 Relationship between the COP and the loading factor (cooling GHPi).

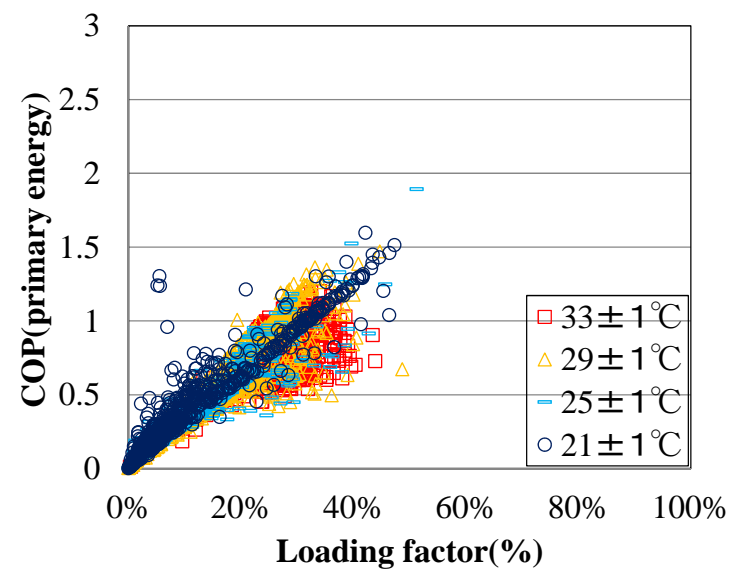

Fig. 12 Relationship between the COP and the loading factor (cooling GHPc).

heating, with the GHPi, the loading factor was also around $40 \%$ or less. But high loading factors of more than $80 \%$ were observed during cooling.

Fig. 13 illustrates the monthly energy efficiency. Which is the ratio of total quantity of air changes of the months divided by the energy consumption of the month' for the GHPc. The maximum energy efficiency was 0.9 during heating. But for the GHPi unit, the maximum energy efficiency was 1.8 during heating. Maximum energy efficiencies were recorded in July. Conversely, minimum energy efficiencies were recorded in April. Energy efficiencies in April were low because the units do not operate often in periods of moderate temperature.

Fig. 14 shows the relationship between energy efficiency and the loading factor. During heating, the 


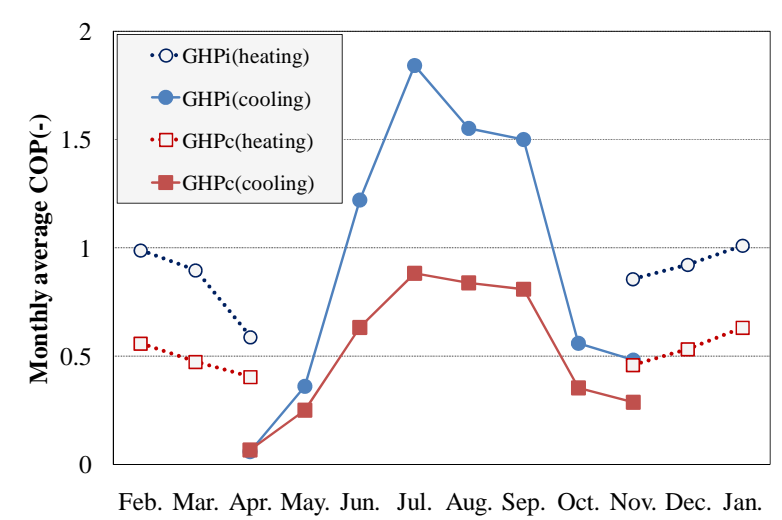

Fig. 13 Quantity of air changes in one year.

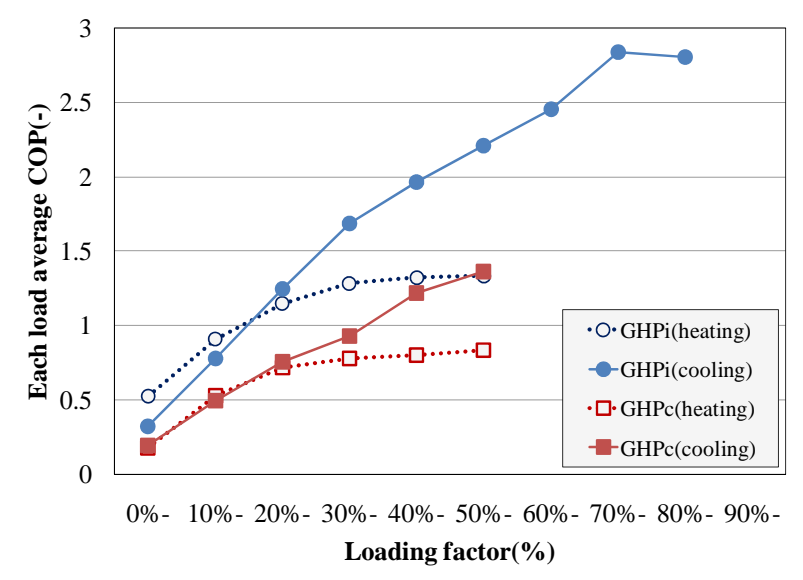

Fig. 14 Each load average COP.

GHPi unit exhibited high loading factors and maximum COP. The highest loading factor was $80 \%$ at a maximum COP of 2.9. Otherwise, the maximum load factor was generally near $50 \%$. When the loading factors were low, there was little difference between the two units, but there was a large difference when loading factors were high.

\section{Conclusions}

The findings in this paper were obtained from actual measurements taken over the course of one year. The air-conditioning abilities were about the same for both
GHPc and GHPi units. However, the GHPc and GHPi units were distinct operation. However, the generated thermal energy of the GHPi was high compared to that of the GHPc. To compare the COP for the two GHP, the authors calculated the monthly average COP. The results show that the COP tends to be high when the demand for heating or cooling is high. However, the COP tends to be higher during cooling. The authors calculated the COP for each loading factor. A high loading factor tends to correlate with a high COP. The COP is greatly affected by load.

\section{References}

[1] T. Nobe, Y. Haga. H. Nakamura, K. Tanaka, M. Kiguchi, Probe insertion method for on-site evaluation of VRF system, Journal of Environmental Engineering Transactions of the Architectural Institute of Japan 668 (2011) 927-933.

[2] K. Yamada, T. Shimizu, T. Nobe, Actual Operation Status and Performance of VRF Systems Determined by Probe Insertion Method, Transactions of the Society of Heating, Air-Conditioning and Sanitary Engineers of Japan 193 (2013) 19-26.

[3] M. Hiraoka, S. Hiromoto, H. Komoda, S. Tabuchi, S. Tanabe, T. Kai, et al., Performance evaluation of multi-split type air-conditioning system and advancing knowledge of HVAC design: Part 1 Performance evaluation of multi-split type air-conditioning system, Transactions of the Society of Heating, Air-Conditioning and Sanitary Engineers of Japan 169 (2011) 13-20.

[4] A. Hosoi, T. Sawachi, H. Miura, Part load characteristic of COP of residential air conditioner based on measurement in arteicial climate chamber: Heating/cooling COP and energy consumption of residential air conditioner part 1, Journal of Environmental Engineering Transactions of the Architectural Institute of Japan 662 (2007) 65-71.

[5] Weather Underground Home Page, http://www.wunderground.com/2012/11/5.

[6] Japanese Industrial Standards B8616, p. 4.

[7] Japanese Industrial Standards C9612, p. 57. 